\title{
Aberrant Carotid Artery in the Middle Ear
}

\author{
Sertaç Yetişer \\ Department of Otorhinolaryngology, Head and Neck Surgery, Anadolu Medical Center, Kocaeli, Turkey
}

Background: Carotid artery abnormality in the middle ear is a rare clinical condition. Recognition of the problems related with this abnormality is important since it may mimic vascular tumors. Any intervention with incomplete evaluation can be fatal.

Case Report: A 23-year-old girl with carotid abnormality and sensorineural hearing loss, unsteadiness and tinnitus is presented. She was followed for 2 months elsewhere assuming that she had Meniere's disease and had previously received some medication for otitis media with effusion.
Conclusion: Tomography and magnetic resonance imaging of the temporal bone are very helpful for visualization of an aberrant carotid in the middle ear. Radiological presentations are the reduced caliber of the aberrant carotid, an absence of cranial opening of carotid canal, tubular coursing along the medial wall of the middle ear in continuity with the horizontal carotid canal, dehiscence of the lateral carotid plate and enlargement of the tympanic canalicus.

Keywords: Aberrant carotid artery, hearing loss, tinnitus
Carotid artery abnormality is an embryologic malformation. Sometimes, its bony wall is absent and the artery is just proximal to the tympanic membrane (1). However, posteriorly displaced carotid artery is a rare vascular abnormality which can present as a red mass behind the eardrum with associated common clinical symptoms. Consequences of an injury to an aberrant carotid are serious and can sometimes be fatal $(2,3)$. Differential diagnosis may be achieved by high resolution computed tomography, angiography and magnetic resonance imaging. We present a patient with carotid abnormality who displayed sensorineural hearing loss, unsteadiness and tinnitus.

\section{CASE PRESENTATION}

A 23-year-old girl was admitted to the outpatient clinic with complaints of hearing loss, unsteadiness, aural fullness and tinnitus in her right ear for the preceding 3 months. Informed consent was obtained from the patient indicating that her data could be used in a scientific article, while en- suring that her anonymity was protected. The procedure was in accordance with the ethical standards of Helsinki declaration and institutional review board. She was followed for 2 months elsewhere assuming that she had Meniere's disease and had previously received some medication for otitis media with effusion. Otoscopic examination demonstrated a bulging whitish mass behind the tympanic membrane (Figure 1). Valsalva maneuver did not reveal any change in size and color. However, some parts of the tympanic membrane appeared to be attached to the mass and did not show any motion synchronous with the pressure changes. Audiometry showed a low frequency sensorineural hearing loss (Figure 2). Tympanogram showed a normal type-A plotting. Nothing was remarkable with the vestibular balance tests. Temporal bone scanning demonstrated an aberrant carotid artery (Figure 3). Magnetic resonance imaging with angiography confirmed the diagnosis (Figure 4). After explaining the situation to the patient, it was decided to follow her with medication. She had some relief of the symptoms during the 6 months period. 


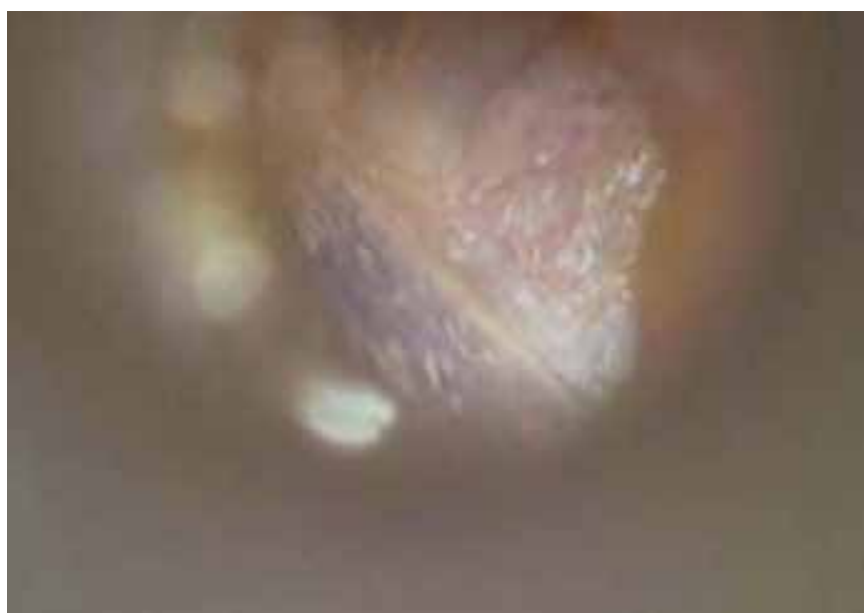

FIG. 1. Otoscopic view of a middle ear mass located slightly anterior to the malleus

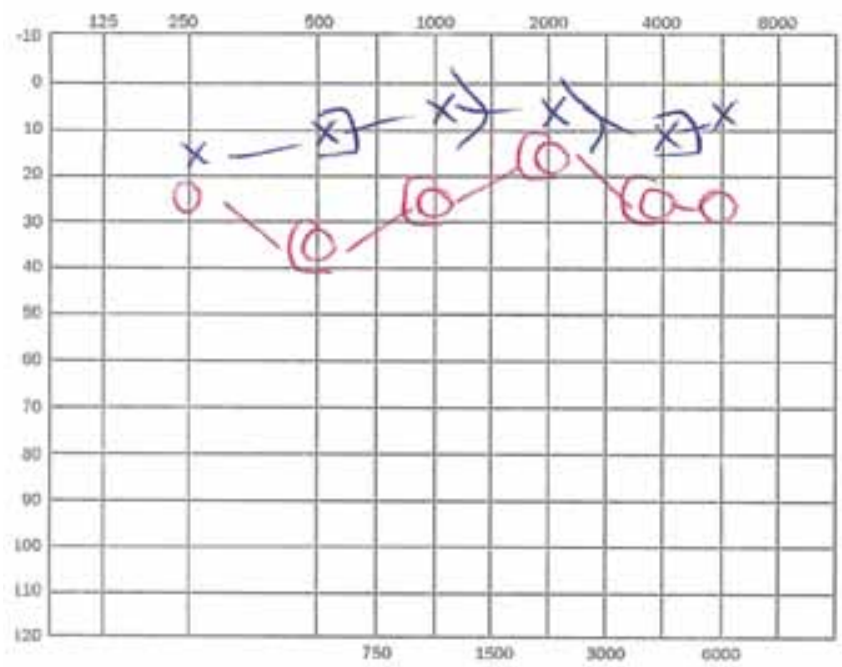

FIG. 2. Low frequency sensorineural hearing loss in the right ear

\section{DISCUSSION}

The internal carotid artery has four portions. The petrous portion has vertical and horizontal pathways along the bony canal through the temporal bone. The carotid enters the temporal bone medial to the styloid process. Its short vertical part is anterior and inferior to the cochlea and is separated from the tympanic cavity by a thin plate of bone. Then, it turns anteromedially and travels in the horizontal plane underneath the Eustachian tube. Later, it enters the cranial fossa via the foramen lacerum. The caroticotympanic branch of the internal carotid artery, which is an embryological remnant of the hyoid artery, leaves the carotid to enter the tympanic cavity above the middle ear. It joins the enlarged inferior tympanic artery at the promontory which is a branch of the ascending pharyngeal
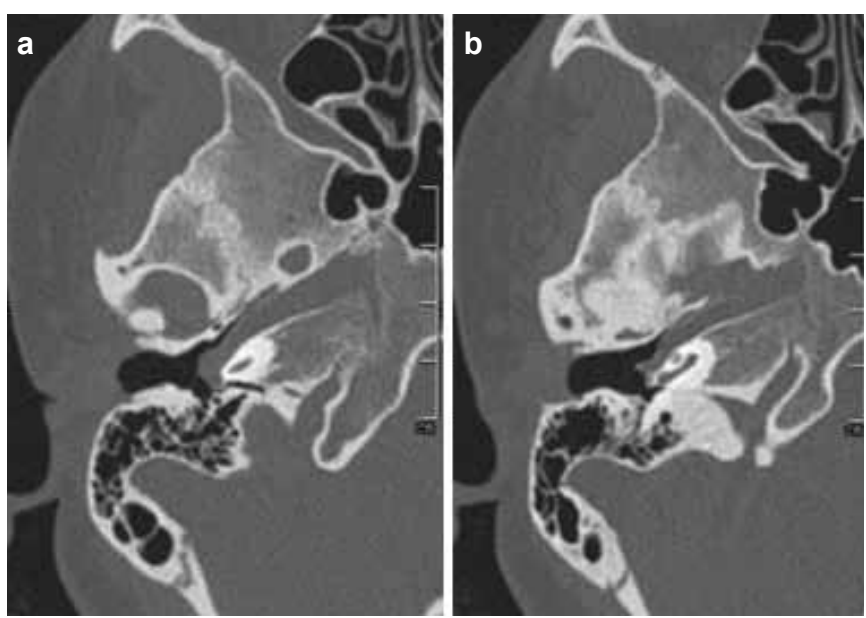

FIG. 3. a, b. Serial axial cut of the temporal bone tomography. Aberrant carotid artery extends to the anterior portion of the middle ear without any bony coverage. It is located over the promontorium (a). Note that the aberrant carotid is in touch with the malleus bone and the ear drum (b)

artery (3). In the absence of the vertical carotid, blood supply to the cerebral region is via the inferior tympanic and caroticotympanic arteries from the horizontal internal carotid artery coursing the tympanic cavity (4). Sometimes, an associated huge stapedial artery, which is actually transient, and embryologic presentation of a derivative of the hyoid artery at the $4^{\text {th }}$ week of fetal life persists as a branch of the aberrant internal carotid artery and leaves the temporal bone through the facial canal to become the middle meningeal artery. In this case, the ipsilateral foramen spinosum is absent (5). One possible assumption is that progressive regression of stapedial and caroticotympanic arteries occurs within the same time frame. A developmental arrest will result in associated persistent stapedial and caroticotympanic abnormalities with the absence of the vertical portion of the carotid.

The incidence of carotid canal dehiscence found at microdissections of the temporal bone ranges from $15 \%$ to $40 \%$ (6). However, this is as low as $1.4 \%$ if studied by computed tomography (7). The incidence of vertical carotid abnormality is obscure, since those patients usually have no other developmental defect in their body. Patients usually have pulsatile tinnitus, which is synchronous with the hearth rate. Conductive type hearing loss is usually related with the fixative mass effect to the tympanic membrane and/or the ossicles or tubal dysfunction (8). Although it is quite rare, a bony dehiscence between the apex of the cochlea and the carotid artery may cause conductive hearing loss due to the "third window effect" (9). However, the mechanism of sensorineural hearing loss is difficult to explain. If the artery takes an aberrant pathway in the tympanic cavity, embryological entrapment may lead to a cochlear anomaly associated with sensorineural hearing loss since the medial wall of the cochlea is made by the carotid ca- 

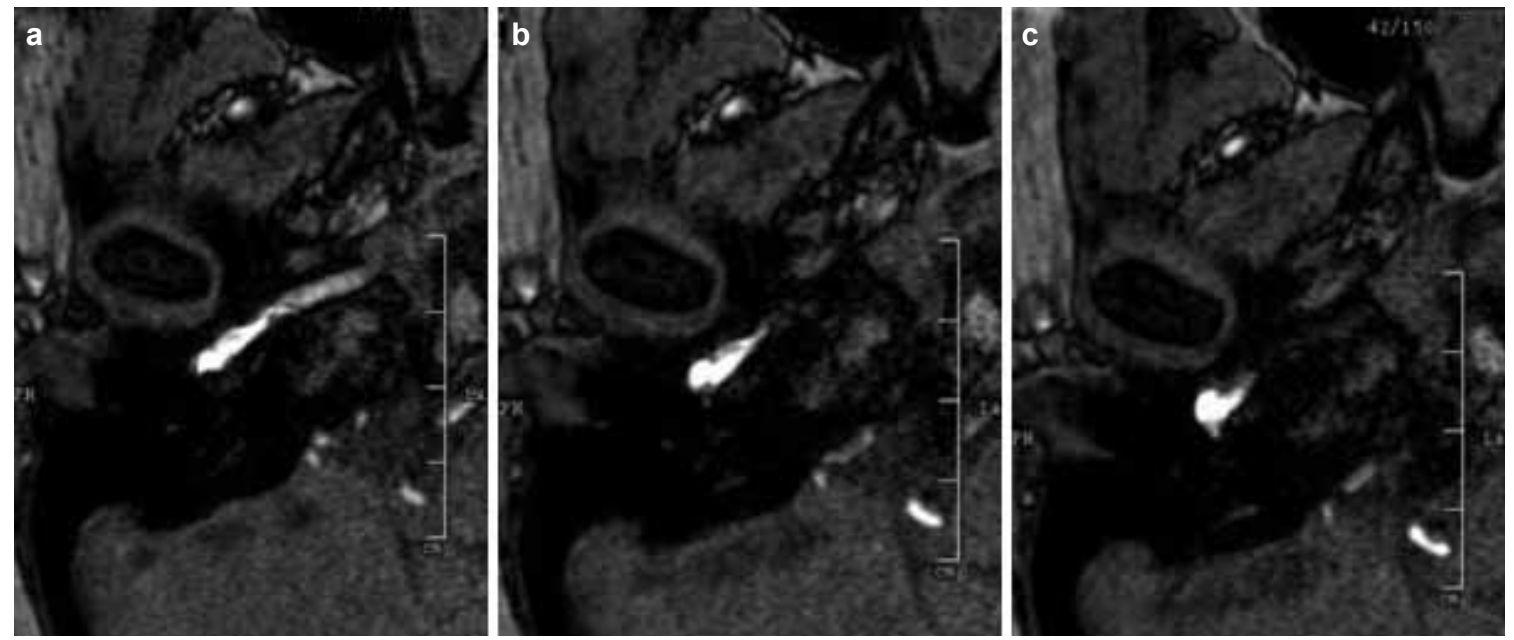

FIG. 4. a-c. Magnetic resonance imaging with angiography shows aberrant carotid artery on the right side (a). In the following cut, aberrant carotid fills the anterior portion of the middle ear (b). In this cut, aberrant carotid resembles a vascular tumor of the middle ear (c)

nal $(9,10)$. It is also likely that an aberrant carotid artery may intervene with the blood flow of the cochlea.

Differential diagnosis prior to attempting surgical manipulation is very important for a pulsating mass behind the tympanic membrane. This dangerous anomaly may mimic otitis media with effusion or acute otitis media, as experienced in our cases. Middle ear tumors, mainly paraganglioma, high jugular bulb, carotid aneurysm, dehiscent, lateralized or aberrant carotid artery should be considered in particular. Tomography and magnetic resonance imaging of the temporal bone are very helpful to ideally visualize the pulsating mass. Radiological presentations for an abnormal course of the carotid artery in the middle ear are the reduced caliber of the aberrant carotid, absence of the cranial opening of the carotid canal, tubular coursing along the medial wall of the middle ear along with the horizontal carotid canal, dehiscence of the lateral carotid plate and enlargement of the tympanic canalicus.

Ethics Committee Approval: This case report was approved by institutional review board.

Informed Consent: An informed consent is provided from the patient.

Peer-review: Externally peer-reviewed.

Acknowledgements: The author would like to thank to all personnel of the department for their valuable help to the patients.

Conflict of Interest: No conflict of interest was declared by the author.

Financial Disclosure: The author declared that this study has received no financial support.

\section{REFERENCES}

1. Hasebe S, Sando I, Orita Y. Proximity of carotid canal wall to tympanic membrane: a human temporal bone study. Laryngoscope 2003;113:802-7. [CrossRef]

2. Jacobsson M, Davidsson A, Hugosson S, Tjellstrom A, Svendsen P. Aberrant intratympanic internal carotid artery: a potentially hazardous anomaly. J Laryngol Otol 1989;103:1202-5. [CrossRef]

3. Soderman M, Moersdorf M, Lysdahl M, Mendel L. Otorrhagia from the aberrant internal carotid artery in the middle ear. Surgical and endovascular aspects. Interv Neuroradiol 1997;30:231-8.

4. Lasjaunias P, Santayo-Vasquez A. Segmental agenesis of the internal carotid artery: angiographic aspects with embryological discussion. Anat Clin 1984;6:133-41. [CrossRef]

5. Roll JD, Urban MA, Larson TC, Gailloud P, Jacob P, Harnsberger HR. Bilateral aberrant internal carotid arteries with bilateral persistent stapedial arteries and bilateral duplicated internal carotid arteries. AJNR Am J Neuroradiol 2003;24:762-5.

6. Moreano EH, Paparella MM, Zelterman D, Goycoolea MV. Prevalence of carotid canal dehiscence in the human middle ear: a report of 1000 temporal bones. Laryngoscope 1994;104:612-8. [CrossRef]

7. Atilla S, Akpek S, Uslu S, Ilgit ET, Isık S. Computed tomographic evaluation of surgically vascular variations related with the temporal bone. Eur J Radiol 1995;20:52-6. [CrossRef]

8. Song YS, Yuan YY, Wang GJ, Dai P, Han DY. Aberrant internal carotid artery causing objective pulsatile tinnitus and conductive hearing loss. Acta Otolaryngol 2012;132:1126-30. [CrossRef]

9. Neyt P, Govaere F, Forton GE. Simultaneous true stapes fixation and bilateral bony dehiscence between the internal carotid artery and the apex of the cochlea: the ultimate pitfall. Otol Neurotol 2011;32:909-13. [CrossRef]

10. Saini J, Kesavadas C, Thomas B, Arvinda HR. Aberrant petrous internal carotid artery with cochlear anomaly. An unusual association. Surg Radiol Anat 2008;230:453-7. [CrossRef] 